\title{
Aspectos característicos da perimólise: uma revisão de literatura entre os anos de
}

\section{0 a 2021}

\author{
Characteristic aspects os perimolysis: a literature review between the years 2010 to 2021 \\ Aspectos característicos de la perimolisis: revisíon de la literatura entre los años 2010 a 2021
}

Júlia Marisa Magalhães de Menezes

ORCID: https://orcid.org/0000-0001-8907-2390 Faculdade Patos de Minas, Brasil

E-mail: Juliamarisa2014@gmail.com

Stéfani Pacheco da Silva

ORCID: https://orcid.org/0000-0003-1111-8963 Faculdade Patos de Minas, Brasil E-mail: estefanypach@gmail.com

Marcelo Dias Moreira de Assis Costa

ORCID: https://orcid.org/0000-0001-9148-3674

Universidade Federal de Uberlândia, Brasil

E-mail: marcelodmac@yahoo.com.br

Eduardo Mendes Moura

ORCID: https://orcid.org/0000-0001-6183-391X

Universidade Federal de Uberlândia, Brasil

E-mail: eduardo.moura@ faculdadepatosdeminas.edu.br

Fernando Nascimento

ORCID: https://orcid.org/0000-0003-3568-3887 Faculdade Patos de Minas, Brasil

E-mail: fernando.nacimento@faculdadepatosdemins.edu.br

Lia Dietrich

ORCID: https://orcid.org/0000-0001-7887-8591 Universidade Federal dos Vales do Jequitinhonha e Mucuri, Brasil E-mail: dietrichlia.ld@gmail.com

\begin{abstract}
Resumo
O objetivo do presente trabalho foi apresentar uma revisão de literatura exploratória sobre o tema perimólise, em virtude da dificuldade na realização do diagnóstico dessa patologia. A revisão de literatura contou com artigos publicados entre os anos de 2010 a 2021, utilizando bases de dados virtuais de saúde, como: Google Acadêmico, SciELO e PubMed. Na Odontologia, desgastes dentários são comumente encontrados em exames clínicos de rotina, apresentam variadas formas e ocorrem em regiões dentais específicas, tendo etiologia multifatorial. A erosão dentária tem sua etiologia de forma extrínseca ou intrínseca, neste último caso, é habitualmente chamada de perimólise. É caracterizada pela desmineralização ácida progressiva de estrutura dental sem envolvimento bacteriano, ocorrendo então, a partir de agentes químicos endógenos diretamente ligados a vômito/arrotos/regurgitação do conteúdo estomacal para o esôfago, que chega a orofaringe e a cavidade oral, afetando o esmalte e a dentina. O diagnóstico pode ser controverso e desafiador, pois pode ser confundido com outros tipos de lesões dentárias, ou passar despercebido em estágios iniciais. É de extrema importância ressaltar a participação do Cirurgião-dentista perante este diagnóstico, que está diretamente ligado à pacientes que apresentam quadros de transtornos alimentares como Bulimia e Anorexia nervosa. Concluiu-se que a perimólise está frequentemente associada a transtornos alimentares, tem progressão lenta, com sinais frequentemente sutis, o que geralmente causa falha no diagnóstico precoce, sendo necessário conhecimento da patologia, e seus fatores etiológicos, para que o profissional seja capaz de realizar tal diagnóstico, conseguindo evitar a progressão para estágios avançados e consequentemente tratamentos mais complexos.
\end{abstract}

Palavras-chave: Erosão dentária; Diagnóstico bucal; Bulimia; Anorexia nervosa.

\begin{abstract}
The aim of the present work was to present an exploratory literature review on the topic of perimolysis, due to the difficulty in diagnosing this pathology. The literature review included articles published between 2010 and 2021, using virtual health databases, such as: Academic Google, SciELO and PubMed. In Dentistry, tooth wear is commonly found in routine clinical examinations, has different forms and occurs in specific dental regions, having a multifactorial etiology. Dental erosion has its etiology in an extrinsic or intrinsic way, in the latter case, it is usually called perimolysis. It is characterized by progressive acid demineralization of tooth structure without bacterial involvement, occurring from endogenous chemical agents directly linked to vomiting/belching/regurgitation of
\end{abstract}


stomach contents into the esophagus, which reaches the oropharynx and oral cavity, affecting the enamel and the dentin. Diagnosis can be controversial and challenging, as it can be confused with other types of dental injuries, or go unnoticed in early stages. It is extremely important to emphasize the participation of the dentist in this diagnosis, which is directly linked to patients with eating disorders such as Bulimia and Anorexia nervosa. It was concluded that perimolysis is often associated with eating disorders, has a slow progression, with often subtle signs, which usually causes failure in early diagnosis, requiring knowledge of the pathology and its etiological factors, so that the professional is able to perform such diagnosis, managing to avoid progression to advanced stages and consequently more complex treatments.

Keywords: Tooth erosion; Oral diagnosis; Bulimia; Anorexia nervosa.

\section{Resumen}

El objetivo del presente trabajo fue presentar una revisión exploratoria de la literatura sobre el tema de la perimolisis, debido a la dificultad en el diagnóstico de esta patología. La revisión de la literatura incluyó artículos publicados entre 2010 y 2021, utilizando bases de datos virtuales de salud, como: Google Academic, SciELO y PubMed. En Odontología, el desgaste de los dientes se encuentra comúnmente en los exámenes clínicos de rutina, tiene diferentes formas y ocurre en regiones dentales específicas, con una etiología multifactorial. La erosión dental tiene su etiología de forma extrínseca o intrínseca, en este último caso se suele denominar perimolisis. Se caracteriza por la desmineralización ácida progresiva de la estructura dental sin afectación bacteriana, producida por agentes químicos endógenos directamente relacionados con los vómitos / eructos / regurgitación del contenido del estómago hacia el esófago, que llega a la orofaringe y la cavidad bucal, afectando el esmalte y la dentina. El diagnóstico puede ser controvertido y desafiante, ya que puede confundirse con otros tipos de lesiones dentales o pasar desapercibido en etapas tempranas. Es sumamente importante destacar la participación del odontólogo en este diagnóstico, que está directamente vinculado a pacientes con trastornos alimentarios como Bulimia y Anorexia nerviosa. Se concluyó que la perimolisis se asocia frecuentemente a trastornos de la conducta alimentaria, tiene una progresión lenta, con signos muchas veces sutiles, lo que suele ocasionar fracaso en el diagnóstico precoz, requiriendo el conocimiento de la patología y sus factores etiológicos, para que el profesional sea capaz de realizar dicho diagnóstico, logrando evitar la progresión a estadios avanzados y consecuentemente tratamientos más complejos.

Palabras clave: Erosión de los dientes; Diagnóstico oral; Bulimia; Anorexia nerviosa.

\section{Introdução}

Dentro da Odontologia, desgastes dentários são comumente encontrados em exames clínicos de rotina, que podem se apresentar de variadas formas e também ocorrer em regiões dentais específicas, sendo sua etiologia classificada de forma multifatorial (Cruz da Silva, Vasconcelos, \& Vasconcelos, 2019).

Estes desgastes nas superfícies dos dentes são comumente classificados em: abrasão (ocorrência de fricção mecânica no dente por agentes externos), atrição (contato de dente com dente), abfração (perda da estrutura dentária cervical provocada por tensões nesta região) e ainda a erosão dentária (perda de estrutura dentária devido a ação de ácidos) onde esta tem sua etiologia de forma extrínseca ou intrínseca, neste último caso, sendo habitualmente chamada de perimólise (Cruz da Silva et al., 2019; Daniel, Ricci, Boeck, Bevilacqua, \& Cerqueira-Leite, 2015; Kulkarni, Rothrock, \& Thompson, 2020; Maltarollo et al., 2020).

Assim, a erosão dentária do tipo perimólise, é caracterizada pela desmineralização ácida progressiva de estrutura dental sem o envolvimento de ação bacteriana, ocorrendo este desgaste por meio de agentes químicos endógenos (Belila et al., 2021; Cruz da Silva et al., 2019; Daniel et al., 2015; Picos et al., 2020).

Em relação a etiologia da erosão dentária, a sua forma extrínseca se dá pelas particularidades do estilo de vida do paciente, como a dieta, ambientes de trabalho (exposição a gases industriais), consumo de alguns medicamentos que alteram a acidez bucal, alcoolismo crônico, misturas de álcool com energéticos, e ainda, estas formas de erosão podem estimular o agravamento dos efeitos já instalados pela forma intrínseca (Canto et al., 2020; Cruz da Silva et al., 2019; Daniel et al., 2015; Kulkarni et al., 2020; Picos et al., 2020).

Os desgastes erosivos por causas extrínsecas são bastantes comuns em relação às variadas mudanças de hábitos alimentares, pois o ser humano cada vez mais faz consumo de alimentos industrializados, que em grande maioria apresentam 
substâncias ácidas como conservantes em sua composição, que contribuem para o aparecimento da perimólise (Daniel et al., 2015).

Já na sua forma intrínseca, esta se encontra diretamente ligada pelo vômito/arrotos/regurgitação do conteúdo estomacal para o esôfago, que consequentemente chega a orofaringe e a cavidade oral, onde estes conteúdos se encontram com um pH muito baixo, afetando o esmalte e a dentina (Belila et al., 2021; Chockattu et al., 2018; Cruz da Silva et al., 2019; Picos et al., 2020).

Diante disso, é importante dizer que estes acontecimentos estão associados a doenças, como a bulimia, distúrbio do refluxo gastroesofágico e a anorexia nervosa (Chockattu et al., 2018; Kulkarni et al., 2020; Milosevic, 2017; Picos et al., 2020).

A aparência clínica da perimólise pode variar bastante, o que vai depender da gravidade, composição e o pH do refluxo, a capacidade de tamponamento da saliva, se o indivíduo escova os dentes imediatamente após o episódio da regurgitação, e o tempo e a frequência de exposição das estruturas dentárias com os ácidos, pois este processo de erosão dentária é lento e ocorre ao longo de anos, não sendo uma surpresa se o diagnóstico for realizado quando já se tem grande prejuízo no sistema mastigatório e na dentição (Milosevic, 2017; Ranjitkar, Smales, \& Kaidonis, 2012).

Classicamente, a perimólise apresenta-se inicialmente com a perda de esmalte e dentina nas superfícies palatinas dos dentes superiores, e em casos de longas datas, a erosão pode chegar a afetar até mesmo a superfície oclusal dos dentes e outras regiões tanto superiores quanto inferiores, levando à problemas de oclusão (Kunde, Mitinguel, Bellato, \& Moreira, 2017; Ranjitkar et al., 2012).

A perda de minerais da superfície dentária pode levar a quadros de dor (sensibilidade), comprometimento estético, e também funcional como já mencionado, sendo esses os motivos para a ocorrência de visitas ao Cirurgião-dentista, entretanto, esta procura pode demorar para ser realizada, e novamente fazer com que o diagnóstico seja tardio, dificultando o tratamento reabilitador, e deixando-o mais oneroso (Daniel et al., 2015).

O diagnóstico desta patologia, pode ser controverso e desafiador, visto que pode ser confundido com outros tipos de lesões dentárias, ou passar despercebido em estágios iniciais, onde a realização de uma anamnese minuciosa, a observação dos possíveis fatores etiológicos, buscando toda a história médica do paciente, seus hábitos alimentares e suas formas de higienização bucal, além da necessidade da realização de um exame clínico detalhado, podem favorecer o diagnóstico precoce (Cruz da Silva et al., 2019; Maltarollo et al., 2020).

Vale reforçar que a dificuldade da realização do diagnóstico pode estar ligado ao paciente não fornecer informações dos possíveis fatores causais por não terem conhecimento da relação dos problemas gástricos com problemas dentais e ainda por este tipo de lesão dentária envolver fatores psicológicos, quando associadas a doenças como a anorexia nervosa e bulimia (Cruz da Silva et al., 2019; Daniel et al., 2015).

A perimólise ainda se encontra como uma patologia de difícil diagnóstico para os Cirurgiões-dentistas, portanto, o diagnóstico e o controle preventivo nos estágios iniciais de tal erosão é uma questão fundamental para evitar terapias restauradoras complexas e onerosas, além de alterações oclusais, sintomatologias dolorosas (sensibilidade dentinária) e comprometimento estético, visto que, os Cirurgiões-dentistas são os profissionais que primeiro tem contato com as características desse tipo de erosão na cavidade bucal, principalmente quando relacionadas a distúrbios psicossomáticos, daí a importância deste trabalho para enfatizar as características clínicas da perimólise e da necessidade de conhecimento dos seus fatores etiológicos e sinais clínicos.

Assim, o presente trabalho possui como objetivo apresentar uma revisão de literatura exploratória sobre o tema perimólise, em virtude das dificuldades que muitos profissionais apresentam em diagnosticar essa patologia. 


\section{Metodologia}

A metodologia abordada para elaborar o artigo em questão consiste em uma revisão de literatura, baseada em bancos de dados virtuais de saúde, como: Google Acadêmico, SciELO e PubMed. Foram selecionados artigos publicados entre os anos 2010 e 2021. Utilizou-se como base palavras chaves: Perimólise, erosão, causas, tratamento e keywords: perimolysis, erosion, causes, treatment.

\section{Revisão de Literatura}

\subsection{Etiologia, manifestações clínicas, e sintomatologia da perimólise}

A Odontologia está em constante evolução, cada dia mais buscando conceitos relacionados a promoção de saúde, aliados ao aumento da expectativa de vida, fazendo com que haja uma crescente busca pela manutenção dos elementos dentais (Catelan, Guedes, \& Santos, 2010). Contudo, mudanças nos hábitos alimentares e também de comportamento podem contribuir para o aparecimento de lesões advindas da perda de estrutura dentária (Catelan et al., 2010).

A perda da estrutura dental, não sendo por motivo carioso, acontece de forma fisiológica ao passar dos anos, entretanto, esta é considerada patológica quando o grau de destruição ocorre de forma excessiva, levando a problemas tanto de natureza funcional e/ou estética, podendo atrapalhar a qualidade de vida do paciente (Catelan et al., 2010; Shitsuka, Ibuki, Nogueira, Mendes, \& Bonecker, 2018).

Existem diferentes formas crônicas de ocorrência da destruição dentária, que levam a perda irreversível da superfície externa do dente, diante disso, a perimólise se classifica como um tipo de erosão dentária (principal causa da perda da estrutura dentária mineralizada), que provoca seus efeitos por meio da destruição química advinda de ácidos de origem endógena, sem a ocorrência de envolvimento bacteriano (Catelan et al., 2010; Chockattu et al., 2018; Kulkarni et al., 2020; Maltarollo et al., 2020; Picos et al., 2020; Ranjitkar et al., 2012; Santana, Silva, Paiva, Cardoso, \& Silva, 2018).

Devido à sua etiologia estar relacionada a fatores intrínsecos, esta patologia comumente se dá pelo retorno do ácido gástrico, que tem um pH muito baixo (menor que 2), em razão de refluxos que podem ser causados voluntariamente (auto induzido) ou de forma involuntária (Catelan et al., 2010; Chockattu et al., 2018; Cruz da Silva et al., 2019; Milosevic, 2017).

É importante dizer que esses episódios de regurgitação podem estar associados a desordens psicossomáticas, sendo de muito interesse o conhecimento da relação entre bulimia nervosa, anorexia nervosa e casos de erosão dental (Catelan et al., 2010; Daniel et al., 2015; Milosevic, 2017; Santana et al., 2018; Santos, Cardoso, Alves, \& Mendonça, 2015).

O período gestacional (ocorrência de refluxos e vômitos principalmente durante o primeiro trimestre), assim como problemas gastrintestinais como a doença do refluxo gastroesofágico (DRGE) que é uma condição médica bastante comum, também podem estar associados (Castilho, Foratori-Junior, \& Sales-Peres, 2019; Catelan et al., 2010; Cruz da Silva et al., 2019; Daniel et al., 2015; Kulkarni et al., 2020; Milosevic, 2017).

Existe grande prevalência de quadros de erosão dentária em pacientes com DRGE (se caracteriza por alterações na mucosa esofágica que resulta em refluxo do conteúdo do estômago para o esôfago) sendo assim, o exame odontológico deve ser introduzido nos protocolos de investigação no paciente com esta doença, para que lesões dentárias sejam diagnosticadas nos seus estágios iniciais, visto que as lesões orais não são percebidas pelos pacientes (podendo passar despercebidas também pelo médico), evitando-se grandes perdas de tecido mineralizado, contudo, a utilização de antiácidos se mostrou eficiente para efeito protetor contra a erosão dentária intrínseca (Correa, Lerco, Cunha, \& Henry, 2012; Kulkarni et al., 2020; Picos et al., 2020; Ranjitkar et al., 2012; Santos, 2016).

Dentro do contexto social moderno, observa-se que a maioria das disfunções gastrintestinais ocorre em pessoas que tentam seguir um padrão de beleza estabelecido pela mídia, que na maioria das vezes, esse ideal de ter um corpo magro leva a 
tais distúrbios psicossomáticos, o que leva a grandes danos sociais e psicológicos (Alves, Paula, Neto, Júnior, \& Cabral, 2018; Daniel et al., 2015; Santos et al., 2015)

A bulimia nervosa se caracteriza por ser um transtorno alimentar reconhecido por vômitos autoinduzidos, atrapalhando o bem estar físico e o funcionamento psicossocial. Tanto a bulimia quanto a DRGE fazem com que o ácido estomacal se acumule na cavidade bucal, por períodos variados de tempo, levando aos efeitos destrutivos na dentição, mas não se limitando apenas à erosão dos dentes (Kulkarni et al., 2020)

A bulimia nervosa se dá pela ingestão de uma grande quantidade de alimentos (de forma excessiva, vontade incontrolável de comer mas com pouca ou nenhuma sensação de prazer), e logo após, surge um sentimento de culpa para tentar controlar o peso, levando a pessoa a fazer uso de laxantes, diuréticos e provocar compulsivamente e intencionalmente o vômito (em casos mais graves estes episódios bulímicos podem ocorrer em dez ou mais vezes durante um período de 24h), onde a manifestação da perimólise nesses casos são clinicamente observados quando o vômito já é estimulado a pelo menos 2 anos (Belila et al., 2021; Daniel et al., 2015; Kunde et al., 2017; Santos et al., 2015).

Já a anorexia nervosa leva a uma aversão do paciente por qualquer tipo de alimento, consequentemente não se alimentando, ou seja, a perda de peso é a partir de dietas rigorosas (grande perda de apetite), que levam a questões psicológicas como sofrimento, preocupação com a alimentação e medo intenso (Daniel et al., 2015; Santos et al., 2015).

Pessoas com esse transtorno apresentam uma distorção da imagem corporal, ou seja, mesmo estes se encontrando abaixo do peso, se veem gordos, e também, a anorexia nervosa pode se apresentar de duas formas, conhecidas como restritiva e a purgativa (Belila et al., 2021; Santos et al., 2015).

A anorexia do tipo restritiva, é quando o paciente segue não mais que uma dieta restritiva e a prática de exercícios intensos, contudo, no tipo purgativa, que apresenta maior interesse para a área odontológica, o paciente come de forma compulsiva e acaba praticando métodos de purgação com medicamentos e também, como a bulimia, provocando o vômito autoinduzido (Alves et al., 2018; Santos et al., 2015).

A perimólise se dá quando o tecido dentário (esmalte e dentina) é exposto ao ataque ácido, ocorrendo uma perda irreversível, localizada (mas podendo ser generalizada), crônica, patológica e indolor (Canto et al., 2020; Cruz da Silva et al., 2019; Santos et al., 2015).

Diante disso, as lesões erosivas intrínsecas aparecem inicialmente nas superfícies palatinas dos dentes superiores, pois a força da regurgitação se inicia a partir da região da faringe, seguindo em direção à cavidade bucal, onde ocorre o impulsionamento do suco gástrico para frente, causando danos nessas superfícies primeiramente, e nas oclusais dos dentes posteriores inferiores posteriormente (Alves et al., 2018; Chockattu et al., 2018; Santos, 2016; Kunde et al., 2017).

Os sinais da perimólise que podem ser facilmente encontrados em um estágio inicial (desde que o exame clínico bucal não seja realizado de forma superficial) incluem um esmalte de superfície brilhante (liso, e vitrificado), com a borda de esmalte próximo a margem gengival intacto, amarelamento da dentina subjacente, e translucidez na borda incisal aumentada (Cruz da Silva et al., 2019; Maltarollo et al., 2020; Santana et al., 2018).

Em casos mais avançados, observa-se bordas fraturadas, perda da dimensão vertical, ocorrência na superfície lingual dos dentes anteriores inferiores (esta costuma estar mais "protegida" pelo constante contato com a saliva), escavação/depressões côncavas das superfícies oclusais dos dentes inferiores, onde os pacientes podem ser assintomáticos. A erosão palatina costuma passar despercebida pelos pacientes, pois estas superfícies não são visíveis, e raramente a exposição pulpar acontece (Cruz da Silva et al., 2018; Daniel et al., 2015; Kunde et al., 2017; Milosevic, 2017; Ranjitkar et al., 2012; Santos, 2016; Shitsuka et al., 2018).

Entretanto, várias são as consequências negativas para os pacientes devido a constante perda de tecido dentário, como o desenvolvimento da hipersensibilidade dentinária, irritação pulpar, restaurações com sobre contorno (ilhas de amálgama), o 
que leva a um tratamento reabilitador longo e dispendioso, além de outros sintomas relacionados a regurgitação na cavidade bucal, como gosto ácido, irritação da mucosa, sensação de queimação, xerostomia, aumento das glândulas parótidas e salivares (Daniel et al., 2015; Kunde et al., 2017; Santos, 2016).

Vale ressaltar a importância da saliva em todo este processo, pois ela é um dos principais elementos responsáveis pela ocorrência de homeostase na cavidade bucal e também no trato digestivo, a saliva contém o potencial para tamponar (neutralizar) o ácido advindo do estômago, diante disso ajuda a combater a perimólise, mas em casos que os pacientes por algum motivo sofrem de hipossalivação e/ou xerostomia, tal patologia se agrava mais rapidamente, podendo ser indicado o uso de saliva artificial (Catelan et al., 2010; Correa et al., 2012; Ranjitkar et al., 2012; Shitsuka et al., 2018).

Importante dizer também que os dentes erodidos com ácido são mais suscetíveis à ocorrência de abrasão, pois sua superfície se torna mais amolecida, devendo-se então ser ensinado ao paciente à não escovar os dentes imediatamente após episódios de regurgitação (Milosevic, 2017).

\subsection{Importância do diagnóstico precoce e tratamento da perimólise}

O grande desafio se tratando dos casos de perimólise é o seu diagnóstico, que muitas vezes acaba passando despercebido pelo profissional Cirurgião-dentista principalmente em seus estágios iniciais, e essa incapacidade pode levar a danos significativos tanto nos dentes como no sistema mastigatório (Daniel et al., 2015; Santos, 2016).

O diagnóstico correto e precoce promove melhores condições de prevenção e formas de tratamento para o paciente, daí a importância do profissional ter conhecimento de todas as suas características (sinais e sintomas), bem como dos fatores etiológicos (Cruz da Silva et al., 2019).

Nos casos que são diagnosticados em estágios já avançados, nem sempre os fatores etiológicos são prontamente identificados, visto que os pacientes podem não saber o que pode ser, ou apresentar a DRGE de forma desconhecida, ou ainda a questão de não estarem dispostos a falar sobre seus transtornos alimentares ao profissional, por terem vergonha ou se sentirem constrangidos, e a partir disso, o tratamento acaba se tornando mais complexo podendo necessitar da reabilitação oral generalizada (Cruz da Silva et al., 2019; Milosevic, 2017).

Importante salientar que a DRGE muitas vezes quando ainda não foi diagnosticada por trazer consigo "reflexos silenciosos", coloca o Cirurgião-dentista como o primeiro profissional da saúde a suspeitar da presença desta doença a partir da observação da erosão dentária presente nos dentes de forma inexplicada pelo paciente, associado a relatos de gosto azedo ou ácido na cavidade bucal e disgeusia (alterações no paladar) (Kunde et al., 2017; Ranjitkar et al., 2012).

Se tratando dos transtornos alimentares como a Bulimia e a Anorexia nervosa, o Cirurgião-dentista também pode vir a ser o primeiro profissional da saúde a ter suspeitas da presença destes distúrbios, pois os pacientes tendem a esconder os episódios das doenças de amigos e da família, daí a importância de estar apto a identificar os sinais e sintomas presentes na cavidade oral, pois o diagnóstico precoce desses distúrbios leva à um prognóstico favorável para o paciente (Belila et al., 2021; Daniel et al., 2015; Santos et al., 2015). Além das manifestações orais, sinais corporais evidentes podem ser percebidos, como machucados no dorso da mão (atrito da mão com os dentes no uso da mão para provocar o vômito), aumento das glândulas salivares (principalmente parótida), perda de peso acentuado, e sinais de desnutrição.

A partir disso, o Cirurgião-dentista precisa também estar preparado para consequente encaminhamento do indivíduo para uma avaliação que será multiprofissional, que muitas vezes se torna uma tarefa difícil motivar os pacientes a procurarem essa ajuda, principalmente psicológica (daí a importância do vínculo profissional-paciente) pois o paciente pode negar a existência da própria doença, e o diagnóstico e tratamento desses transtornos requerem uma abordagem transdisciplinar, com o Cirurgião-dentista dentro desta equipe (Daniel et al., 2015; Santos et al., 2015) 
Para o diagnóstico precoce da perimólise, muito importante também a realização de uma ótima anamnese, dando ênfase não só as questões odontológicas do paciente mas também de toda a sua história médica, como questões médicas que podem predispor o paciente a erosão dental, uso de medicamentos que podem provocar a diminuição do fluxo salivar (que agrava o efeito erosivo nos dentes), e também muita atenção em aspectos que levam ao diagnóstico diferencial de lesões erosivas causadas por fatores exógenos de fatores endógenos que é a observação das superfícies que estão sendo ou que foram afetadas pela desmineralização (Catelan et al., 2010; Cruz da Silva et al., 2019).

Casos de ocorrência da desmineralização nas superfícies palatinas tanto dos dentes superiores anteriores quanto na região lingual dos inferiores anteriores, levam a sugestão diagnóstica de que o ácido é de origem endógena, já a erosão associada a superfícies vestibulares dos dentes anteriores leva a erosão por fatores exógenos como exemplo alimentação ácida (Cruz da Silva et al., 2019).

Nos últimos anos houve significantes alterações no estilo de vida das pessoas, e também na qualidade da alimentação da população, tais como o aumento do consumo de bebidas industrializadas, como sucos e refrigerantes, e também bebidas estimulantes como isotônicas (muito consumida por atletas) e energéticos, sendo consumidos diariamente, o que promove acentuado aumento de lesões não cariosas nos dentes (Moretto, Pedra, Carvalho, Silva, \& Fernandes, 2017).

Tais bebidas possuem um $\mathrm{pH}$ abaixo de 5, ou seja, abaixo do $\mathrm{pH}$ crítico para dissolver o esmalte $(\mathrm{pH}=5,5)$, e possuem uma grande variedade de componentes ácidos (como o ácido cítrico), que promovem a desmineralização do esmalte dentário. O ácido cítrico, além do seu pH reduzido tem uma propriedade quelante em relação ao cálcio, assim, além de promover a desmineralização compromete o processo de remineralização (Fontineli, \& Vieira, 2019; Moretto et al., 2017)

Assim, o consumo crônico e frequente de bebidas ácidas associado à escovação mecânica imediata, abrasivos contidos nos dentifrícios, favorece a instalação ou o agravamento de lesões como a erosão/atrição/abrasão já existentes (Fontineli, \& Vieira, 2019; Moretto et al., 2017).

Contudo, partir do momento que se diagnostica a perimólise, pode-se começar a atuar sobre os fatores causais da lesão com um sentido de educação e conscientização do indivíduo, impedindo sua formação ou a sua progressão, buscando assim, restituir a função, forma e estética para o paciente (Catelan et al., 2010).

Sobre o tratamento da perimólise, a conduta clínica dependerá do grau de severidade da erosão nos dentes, podendo ser desde aplicações tópicas de flúor semanais ou agentes dessensibilizantes, até tratamentos reabilitadores (resinas, coroas protéticas, endodontia) quando da perda de estruturas dentais, podendo ocorrer danos funcionais mais extensos (como a perda da dimensão vertical) e/ou estéticos (Catelan et al., 2010; El Achkar, Back-Brito, \& Koga-Ito, 2012).

É importante salientar que as intervenções odontológicas devem ser realizadas de forma integrada ao tratamento realizado pelos outros profissionais da saúde, visto que, tal erosão está relacionada principalmente aos transtornos alimentares, que exigem um tratamento multidisciplinar (Kunde et al., 2017; Santos et al., 2015)

Assim, o tratamento odontológico vai consistir de fases, como: tratamento das urgências, que seria o alívio da dor e melhora da estética (a dor pode ser controlada com a colocação de cimentos de ionômero de vidro sobre os dentes com dentina exposta, ou ainda vernizes fluoretados), terapia causal, terapia reabilitadora e a manutenção preventiva (El achkar et al., 2012; Kunde et al., 2017; Santos et al., 2015).

Inicialmente, o Cirurgião-dentista deve fornecer informações importantes sobre a higienização bucal, como não escovar os dentes imediatamente após os episódios (esperar pelo menos 20 minutos), pois como já mencionado, os dentes sofrendo ataque ácido são mais susceptíveis à abrasão, bem como fazer a utilização de escovas extra macias, pasta de dente menos abrasivas e com maior concentração de flúor (5000ppm) ou com produtos naturais, utilização de antiácidos após o vômito, estímulo do fluxo salivar com chicletes sem açúcar, para os pacientes que apresentam xerostomia, orientação para a utilização de soluções para bochecho com água e bicarbonato de sódio, ou bochechos com fluoreto de sódio à 0,05\% (para 
neutralização do pH ácido da boca), aplicações tópicas de flúor ou terapias dessensibilizantes (Chimbinha, Jácome, Silva, Barreto, \& Costa, 2019; Daniel et al., 2015; Milosevic, 2017; Santana et al., 2018; El achkar et al., 2012).

$\mathrm{O}$ aconselhamento dietético também deve ser realizado, para que o paciente evite alimentos muito ácidos, desde sucos ou frutas cítricas, produtos com cafeína, energéticos e refrigerantes (El achkar et al., 2012).

A perda da estrutura dental é irreversível, mesmo após a cura da desordem alimentar, daí é importante dizer que trabalhos restauradores definitivos deverão ser realizados somente quando o paciente obter controle do hábito de purgação (Chimbinha et al., 2019; El achkar et al., 2012; Kunde et al., 2017; Santos et al., 2015).

Os tratamentos reabilitadores, podem ser realizados com restaurações diretas em resina, ou com cimento de ionômero de vidro, e também com tratamentos reabilitadores indiretos, como coroas totais, laminados cerâmicos, inlays e onlays, tais opções vão depender da complexidade de cada caso, valendo dizer que na ocorrência de casos severos de desgastes, tratamentos endodônticos podem ser necessários (Catelan et al., 2010; Cruz da Silva et al., 2019; Daniel et al., 2015).

Juntamente com a terapia restauradora, a terapia preventiva também deve ser realizada, como o uso terapêutico de flúor, bochechos, o reforço das orientações de higiene oral para manutenção do tratamento realizado, e também a conscientização dos pacientes que induzem os episódios de vômito sobre os riscos na cavidade bucal decorrente de tal prática (Daniel et al., 2015; El achkar et al., 2012).

O Cirurgião-dentista tem um papel fundamental no diagnóstico precoce de transtornos alimentares, pois pode ser o primeiro profissional de saúde a ter contato com sinais e sintomas na cavidade oral, que juntamente com a realização de excelente anamnese, exames tanto intra como extra orais, poderá dar início ao tratamento das lesões bucais e encaminhar o paciente para tratamento multidisciplinar, contribuindo para o tratamento do transtorno alimentar como um todo (Alves et al., 2018; Ferreira, \& Macri, 2021; Kunde et al., 2017; Maciel, \& Cé, 2017; Soares, 2018).

Entretanto, é válido dizer que pouco é o conhecimento dos Cirurgiões-dentistas sobre sinais e sintomas de transtornos alimentares, o que pode comprometer o diagnóstico precoce, sendo de grande importância que os profissionais se aprofundem em conhecimentos sobre os transtornos alimentares, com a intenção de promover diagnósticos rápidos e precisos (Maciel, \& Cé, 2017; Soares, 2018).

A erosão dentária é a manifestação oral mais comum da regurgitação na forma crônica, típica de distúrbios alimentares. No entanto, tal manifestação não é comum em processos agudos. A erosão dentária, em seus estágios iniciais, pode passar despercebida, daí a necessidade de realização de minucioso exame clínico, com a superfície dental seca, limpa, com excelente iluminação, uma vez que tais cuidados podem facilitar a identificação e diagnóstico das lesões (Alves, Lucena, Araujo, \& Carvalho, 2012; Alves et al., 2018; Chimbinha et al., 2019; Ferreira, \& Macri, 2021; Kunde et al., 2017; Soares, 2018).

Vale ressaltar que durante a realização de anamnese, na suspeita de presença de transtornos alimentares, o Cirurgiãodentista deve abordar o paciente de certa forma que ele se sinta seguro em relatar (pois podem se sentir envergonhados), perguntando sobre seus hábitos alimentares, possibilidade de problemas gastrointestinais, induzi-lo a necessidade de procurar um médico, não realizando diretamente um interrogatório sobre algum transtorno, e se necessário, falar sobre o transtorno com cautela, demonstrando habilidade de apresentar o assunto (El achkar et al., 2012; Soares, 2018).

Em seu trabalho, (Daniel et al., 2015) relata um caso clínico de paciente que procurou atendimento odontológico por queixa principal de "desgaste e sensibilidade dentária", e que com a associação de exame clínico e anamnese foi diagnosticada a perimólise. A condição de negação ou a não aceitação da doença foi muito evidente na paciente do caso clínico, que somente após ter certo grau de familiaridade com o Cirurgião-dentista durante as consultas odontológicas, acabou contando que provocava vômitos diariamente após comer. Segundo a paciente, ela nem havia relatado tal condição ao psiquiatra que a acompanhava por depressão. Então, esses dados associados às características clínicas da lesão, permitiram o diagnóstico de 
perimólise, confirmando a questão de o Cirurgião-dentista ser o primeiro profissional a se deparar com as características da perimólise relacionadas a distúrbios psicossomáticos.

Entretanto nem todos as pessoas apresentam a perimólise, visto que, sua gravidade e progressão estão relacionadas a fatores como tempo e duração da doença, a frequência, quantidade de saliva para a neutralização do ácido e hábitos de higiene (Alves et al., 2012; Alves et al., 2018; Chimbinha et al., 2019; Soares, 2018).

Nestes casos iniciais, é indispensável o conhecimento de demais sinais e sintomas que podem estar presentes na cavidade bucal que indicam presença de transtornos alimentares, visto que, podem ter repercussão não só nos dentes mas também nos demais tecidos bucais, como o aumento de volume das glândulas salivares principalmente nas parótidas, eritema do palato, gengivas e faringe, xerostomia, gengivite, as papilas interdentais hipertrofiadas, palato mole ulcerado pela utilização de objetos para provocar os vômitos, queilite angular, candidíase oral e também no corpo do paciente, como o sinal de Russel (escoriações dorsais da pele, das mãos ou calo na ponta do dedo utilizado para provocar o vômito), apatia, fraqueza, flutuação de peso, palpitações, dificuldade de concentração, entre outros (Alves et al., 2018; Chimbinha et al., 2019; El achkar et al., 2012; Ferreira, \& Macri, 2021; Maciel, \& Cé, 2017; Soares, 2018).

\subsection{Possíveis causas para o aumento de casos de perimólise}

Segundo (El Achkar et al., 2012), o número de casos de transtornos alimentares vem aumentando consideravelmente ao longo dos anos, estimulado pela sociedade moderna que elege a magreza um tipo de símbolo de sucesso e beleza.

A ideal imagem de beleza passada e enfatizada pela mídia, tem gerado nas pessoas obsessão e preocupação para a obtenção de corpos magros, com isso a sociedade contemporânea apresenta números crescentes do aparecimento de transtornos alimentares nas pessoas, como já mencionado a Bulimia e a Anorexia Nervosa, que causam sérios riscos a saúde dos sujeitos que as desenvolvem (Kunde et al., 2017; Maciel, \& Cé, 2017; Maltarollo et al., 2020: Patterson-Norrie, Ramjan, Sousa, Sank, \& George, 2020; Soares, 2018).

A juventude é um período propenso para a ocorrência de insatisfação com o próprio corpo, ocorrem várias mudanças tanto físicas, como sociais e psicológicas (que podem influenciar no comportamento alimentar da pessoa) além da forte influência dos meios de comunicação que fazem os jovens encontrarem-se na constante busca de padrões ideais de beleza, sendo assim, este grupo da população apresenta maiores chances em desenvolver algum tipo de transtorno, pois ainda não possuem critérios e valores próprios para conseguirem evitar tais modelos estéticos vigentes (Bittar, \& Soares, 2020; Ferreira, \& Macri, 2021; Kunde et al., 2017; Maciel, \& Cé, 2017).

A idade média de tal ocorrência geralmente está entre os 17 a 25 anos, contudo, podendo ocorrer desde o início da adolescência, com prevalência no sexo feminino, e vale salientar que a maioria dos padrões de beleza idealizados é impróprio ou até mesmo impossível de ser seguido pela maioria da população, trazendo angústia e frustação às pessoas( Bittar, \& Soares, 2020; Ferreira, \& Macri, 2021; Maciel, \& Cé, 2017; Soares, 2018).

A partir disso, os transtornos alimentares demonstram também efeitos prejudiciais a saúde bucal, como já mencionado, tendo como principal manifestação, a erosão dentária do tipo perimólise, que se torna presente na vida de pessoas que desenvolvem transtornos alimentares, sendo importante dizer que tais desgastes aumentam a insatisfação corporal, promovendo maior declínio da autoestima, bem como da qualidade de vida e da interação nos relacionamentos sociais (Patterson-Norrie et al., 2020).

\section{Discussão}

A perimólise é a perda irreversível de tecido dental que se caracteriza pela ação de ácidos de origem endógena na cavidade bucal, sendo de extrema importância ressaltar a participação do Cirurgião-dentista perante o diagnóstico desta 
patologia, que está diretamente ligada à pacientes que se encontram em quadros de transtornos alimentares como a Bulimia e Anorexia nervosa (Ferreira, \& Macri, 2021).

Nesta revisão narrativa da literatura, baseado nos artigos pesquisados, observa-se a dificuldade do diagnóstico precoce da perimólise, visto que apresenta variadas características clínicas e que inicialmente, podem passar despercebidas pelo profissional, que, tem grande probabilidade de ser um dos primeiros profissionais a ter contato com suas características e possivelmente diagnosticar sua etiologia, e que quando mais cedo a identificação e deteç̧ão dos transtornos alimentares em adolescentes e crianças, melhor será o prognóstico para esses pacientes (Alves et al., 2012).

Em uma pesquisa realizada por (Maciel, \& Cé, 2017), sobre o conhecimento dos Cirurgiões-dentistas sobre as manifestações orais em pacientes portadores de transtornos alimentares, contando com um total de 100 profissionais, que por meio de questionários para verificação de tal conhecimento, foi constatado que, sobre o conhecimento a respeito da Anorexia Nervosa e Bulimia Nervosa, $94 \%$ dos participantes possuíam pouco conhecimento, $4 \%$ possuíam muito conhecimento, e $2 \%$ não tinham conhecimento em relação a esses dois transtornos alimentares.

E já em relação ao diagnóstico de pacientes com transtornos alimentares, $74 \%$ dos participantes indicaram que talvez saberiam diagnosticar, 22\% indicaram que sim, que saberiam diagnosticar, e 4\% indicaram que não saberiam diagnosticar.

Concluiu-se então que o conhecimento teórico dos Cirurgiões-dentistas sobre tais transtornos compromete o diagnóstico das alterações encontradas tanto nos tecidos moles como nos tecidos duros da cavidade oral.

Assim, considerando que os transtornos alimentares podem repercutir tanto nos dentes, bem como nos tecidos bucais, o conhecimento sobre essas desordens tem sido de interesse para os profissionais odontólogos (Maciel, \& Cé, 2017).

Existe um consenso nos trabalhos estudados desta revisão, que a associação de uma eficiente anamnese e as informações conseguidas após a realização de minucioso exame clínico, favorecem o diagnóstico precoce, e que na suspeita da presença de algum transtorno o Cirurgião-dentista tenha a habilidade de falar sobre o assunto de forma que não deixe o paciente constrangido, mas sim que conquiste sua confiança para que ele fale sobre seus hábitos, problemas gastrointestinais, e emocionais, e assim, o profissional propor um encaminhamento aos profissionais competentes, e tratamento multidisciplinar, .

Diante de tudo, vale ressaltar que os objetivos desta revisão foram alcançados, possibilitando maior agregação de conhecimento para os Cirurgiões-dentistas que não tem grande entendimento sobre os transtornos alimentares bem como das suas manifestações na cavidade bucal.

Entretanto, mais estudos abrangendo tais assuntos na atualidade se fazem necessários, para a obtenção de maior compreensão das características bucais associadas aos transtornos e formas de abordagem ao paciente.

\section{Considerações Finais}

Com base nas evidências disponíveis, a erosão dental do tipo perimólise está frequentemente associada a transtornos alimentares, tem uma progressão de forma lenta, com sinais frequentemente sutis, o que geralmente causa falha no diagnóstico precoce, fazendo com que passe despercebida.

Assim, para que seu diagnóstico em estágios iniciais seja realizado, é necessário conhecimento da patologia e também seus fatores etiológicos, realização de excelente exame clínico que juntamente com dados da anamnese, o profissional seja capaz de realizar tal diagnóstico, conseguindo evitar a progressão para estágios mais avançados e consequentemente tratamentos mais complexos, bem como, saber realizar o manejo do paciente frente a percepção da presença de transtornos alimentares. 


\section{Referências}

Alves, K. C., Paula, P. N. R. de., Neto, A. J. F., Júnior, P. C. S., \& Cabral, L. C. (2018). Manifestações orais dos transtornos alimentares: revisão de literatura. Demetra, 13 (4), 783-792. 10.12957/demetra.2018.31360. https://www.e-publicacoes.uerj.br/index.php/demetra/article/view/31360.

Alves, M. do S. C., Lucena, S. C. de., Araujo, S. G., \& Carvalho, A. L. A. (2012). Diagnóstico clínico e protocolo de tratamento do desgaste dental não fisiológico na sociedade contemporânea. Odontol. Clín.-Cient., Recife, $11 \quad$ (3), $247-251 . \quad$ http://revodonto.bvsalud.org/ scielo.php?script=sci_arttext\&pid=S1677-38882012000300014\#: :text=O\%20desgaste\%20dental\%20que\%20ocorre,estar\% 20relacionados\%20a\% 20desga ste\% 20excessivo.

Belila, N. de M., Martins, R. J., Garbin, A. J. I., Moimaz, S. A. S., Chaves Neto, A. H., \& Garbin, C. A. S. (2021). Análise da saúde bucal e parâmetros bioquímicos salivares de mulheres com anorexia e bulimia nervosa. Research, Society and Developmen, 10 (3), e8710312971. https://doi.org/10.33448/rsdv10i3.12971. https://rsdjournal.org/index.php/rsd/article/view/12971.

Bittar, C., \& Soares, A. (2020). Mídia e comportamento alimentar na adolescência. Cadernos Brasileiros de Terapia Ocupacional., 28 (1), 291-308. https://doi.org/10.4322/2526-8910.ctoAR1920. https://www.scielo.br/j/cadbto/a/mfTpzZ6F3YhywBGx5tVLkgx/abstract/?lang=pt.

Canto, F. M. T. et al. (2020). Comparative Effect of Calcium Mesoporous Silica Versus Calcium and/or Fluoride Products Against Dental Erosion. Braz Dent J., 31 (2), 164-170. 10.1590/0103-6440202002557. https://pubmed.ncbi.nlm.nih.gov/32556016/.

Castilho, A. V. S. S., Foratori-Junior, G. A., \& Sales-Peres, S. H. de C. (2019). Impacto da cirurgia bariátrica no refluxo gastroesofágico e no desgaste dental: uma revisão sistemática. ABCD, arq. bras. cir. dig., 32 (4), e1466. https://doi.org/10.1590/0102-672020190001e1466. http://www.scielo.br/s cielo.php?script=sci_arttext\&pid=S0102-67202019000400501\&lng=en\&nrm=iso.

Catelan, A., Guedes, A. P. A., Santos, P. H. (2010). Erosão dental e suas implicações sobre a saúde bucal. RFO UPF. 15 (1). http://revodonto.bvsalud.org/scielo.php?pid=S1413-40122010000100015\&script=sci_arttext.

Chimbinha, I. G. M., Jácome, A. do N., Silva, G. G. da., Barreto, M. J. R., \& Costa, I. C. C. (2019). Transtornos alimentares e manifestações orais em adolescentes. Revista Ciência Plural, 5 (3), 1-20. https://periodicos.ufrn.br/rcp/article/view/19204.

Chockattu, S.J. et al. (2018). Tratamento da erosão dentária induzida por refluxo gastroesofágico com estratificação direta de resina composta auxiliada por matriz flexível de tala. Odontologia restauradora e endodôntica, $43 \quad$ (1). https://www.ncbi.nlm.nih.gov/pmc/articles/PMC5816990/,

Correa, M. C. C. S. F., Lerco, M. M., Cunha, M. L. R. S. da., \& Henry, M. A. C. A. (2012). Salivary parameters and teeth erosions in patients with gastroesophageal reflux disease. Arq. Gastroenterol. São Paulo, 49 (3), 214-218. https://doi.org/10.1590/S0004-28032012000300009. http://www.scielo.br/scielo.php?script=sci_arttext\&pid=S0004-28032012000300009\&lng=en\&nrm=iso.

Cruz da Silva, E. T., Vasconcelos, R. G., \& Vasconcelos, M. G. (2019). Lesões cervicais não cariosas: considerações etiológicas, clínicas e terapêuticas. Revista cubana de estomatologia. Ciudad de La Habana, 56 (4): e1998. http://scielo.sld.cu/scielo.php?script=sci_arttext\&pid=S0034$75072019000400011 \& \operatorname{lng}=\mathrm{es} \& \mathrm{nrm}=\mathrm{iso}$.

Daniel, C. P., Ricci, H. A., Boeck, E. M., Bevilacqua, F. M., \& Cerqueira-Leite, J. B. B. (2015). Perimolysis: case report. RGO, Rev. Gaúch. Odontol., Campinas , 63 (2), 213-218. https://doi.org/10.1590/1981-863720150002000122397. http://www.scielo.br/scielo.php?script=sci_arttext\&pid=S1981$86372015000200213 \& \operatorname{lng}=$ en\&nrm=iso.

El Achkar, V. N. R., Back-Brito, G. N., \& Koga-Ito, C. Y. (2012). Saúde bucal de pacientes com transtornos alimentares: o marcante papel do cirurgiãodentista. Revista de Odontologia da Universidade Cidade de São Paulo, 24 (1), 51-56. ISSN 1983-5183. https://doi.org/10.26843/ro_unicid.v24i1.355. Disponível em: http://publicacoes.unicid.edu.br/index.php/revistadaodontologia/article/view/355.

Ferreira, T. E., \& Macri, R. T. (2021). Manifestações clínicas orais de pacientes com bulimia e a importância do cirurgião dentista: uma revisão bibliográfica. Revista Interciência - IMES, Catanduva, 1 (5). https://www.fafica.br/revista/index.php/interciencia/article/view/251.

Fontineli, J. G., \& Vieira, L. E. C. (2019). Bebidas Isotônicas Associadas Com A Ocorrência De Lesão De Cárie E Erosão Dental: revisão integrativa da literatura. Trabalho de Conclusão de Curso - Centro Universitário Uninovafapi, Teresina. https://assets.uninovafapi.edu.br/arquivos/old/arquivos_aca demicos/re positorio_Biblioteca/odontologia/20191/BEBIDAS\%20ISOT\%C3\%94NICAS\%20ASSOCIADAS\%20COM\%20A\%20OCORR\%C3 \%8ANCIA\%20DE\%20LES\%C3\%830\%20DE\%20C\%C3\%81RIE\%20E\%20EROS\%C3\%83O\%20DENTAL\%20revis\%C3\%A3o\%20integra2.pdf.

Kulkarni, A., Rothrock, J., \& Thompson, J. (2020). Impact of Gastric Acid Induced Surface Changes on Mechanical Behavior and Optical Characteristics of Dental Ceramics. J Prosthodont., 29 (3), 207-218. 10.1111/jopr.12716. https://pubmed.ncbi.nlm.nih.gov/29333707/.

Kunde, F. R., Mitinguel, L. H., Bellato, A., \& Moreira, M. A. (2017). Perimólise em pacientes portadores de bulimia nervosa do tipo purgativa: revisão de literatura. Revista de Divulgação Científica da ULBRA Torres, 13 (3). http://www.periodicos.ulbra.br/index.php/ci/article/view/4004.

Maciel, N. L., \& CÉ, L. C. (2017). Conhecimento dos cirurgiões dentistas sobre manifestações orais em pacientes portadores de transtornos alimentares. Journal of Oral Investigations, 6 (1). https://seer.imed.edu.br/index.php/JOI/article/view/1026.

Maltarollo, T. H., Pedron, I. G., Medeiros, J. M. F., Kubo, H., Martins, J. L., \& Shitsuka, C. (2020). A erosão dentária é um problema !. Pesquisa, Sociedade e Desenvolvimento, 9 (3), e168932723. https://doi.org/10.33448/rsd-v9i3.2723. https://rsdjournal.org/index.php/rsd/article/view/2723.

Milosevic, A. (2017). Acid Erosion: An Increasingly Relevant Dental Problem. Risk Factors, Management and Restoration. Prim Dent J., 6 (1), $37-45$. 10.1177/205016841700600105. https://pubmed.ncbi.nlm.nih.gov/28376962/.

Moretto, M. J., Pedra, F. P. G., Carvalho, M. O., Silva, J. P. P., \& Fernandes, S. L. (2017). Erosão dentária provocada por bebidas ácidas. Revista Saúde Multidisciplinar, 4 (1). http://revistas.famp.edu.br/revistasaudemultidisciplinar/article/view/47. 
Research, Society and Development, v. 10, n. 14, e03101421665, 2021

(CC BY 4.0) | ISSN 2525-3409 | DOI: http://dx.doi.org/10.33448/rsd-v10i14.21665

Patterson-Norrie, T., Ramjan, L., Sousa, M. S., Sank, L., \& George, A. (2020). Eating disorders and oral health: a scoping review on the role of dietitians. Journal of Eating Disorders, 8 (49). https://link.springer.com/article/10.1186/s40337-020-00325-0.

Picos, A. et al. (2020). Factors associated with dental erosions in gastroesophageal reflux disease: a cross-sectional study in patients with heartburn. Med Pharm Rep., 93 (1), 23-29. 10.15386/mpr-1332. https://pubmed.ncbi.nlm.nih.gov/32133443/.

Ranjitkar, S., Smales, R. J., \& Kaidonis, J. A. (2012). Oral manifestations of gastroesophageal reflux disease. J Gastroenterol Hepatol., 27 (1), 21-7. doi: 10.1111/j.1440-1746.2011.06945.x. https://pubmed.ncbi.nlm.nih.gov/22004279/.

Santana, N. M. S., Silva, D. R., Paiva, P. R. R., Cardoso, A. M. R., \& Silva, A. C. B. (2018). Prevalência de erosão dentária e fatores associados em uma população de escolares. Rev. odontol. UNESP, Araraquara, 47 (3), 155-160. https://doi.org/10.1590/1807-2577.03518. http://www.scielo.br/ scielo.php?script=sci_arttext\&pid=S1807-25772018000300155\&lng=en\&nrm=iso.

Santos, F. D. G., Cardoso, I. C. G., Alves, D. C. B., \& Mendonça, S. M. S. (2015). Anorexia nervosa e bulimia nervosa: alterações bucais e importância do cirurgião-dentista na abordagem multiprofissional. Revista de Odontologia da Universidade Cidade de São Paulo, 27 (1), 33. doi:https://doi.org/10.26843/ro_unicid.v27i1.242. Disponível em: http://publicacoes.unicid.edu.br/index.php/revistadaodontologia/article/view/242.

Santos, J. A. dos. (2016). Manifestações orais em pacientes com a doença do refluxo gastroesofágico: estudo caso-controle. Tese - Universidade Federal de Pernanbuco, Recife. https://repositorio.ufpe.br/handle/123456789/25973.

Shitsuka, C., Ibuki, F. K., Nogueira, F. N., Mendes, F. M., \& Bonecker, M. (2018). Avaliação do estresse oxidativo da saliva de crianças com erosão dentária. Einstein (São Paulo), São Paulo, 16 (2), eAO4203. https://doi.org/10.1590/s1679-45082018ao4203. http://www.scielo.br/scielo.php?script=sc i_arttext\&pid=S1679-45082018000200208\&lng=en\&nrm=iso.

Soares, M. A. (2018). A importância do cirurgião-dentista no diagnóstico da bulimia. Trabalho de Conclusão de Curso - Universidade do Sul de Santa Catarina, Tubarão. https://www.riuni.unisul.br/handle/12345/7298. 